\title{
Development of Novel Crystal Forms of Metaxalone for Solubility Enhancement
}

\author{
M. S. AZIZ, CHITRA GUPTA AND L. K. TYAGI* \\ Lloyd Institute of Management and Technology (Pharm.), Plot No.11, Knowledge Park-II, Greater Noida, Uttar Pradesh \\ 201306, India
}

Aziz et al.: Development of novel crystal forms of metaxalone

Metaxalone is oxazolidin-2-one derivative, biopharmaceutics classification system class II drug, available in the market as a central muscle relaxant drug, used to alleviate discomforts associated with severe, painful musculoskeletal conditions. The major drawbacks associated with formulation of solid oral dosage forms of metaxalone are its poor aqueous solubility, poor flow property and low compressibility. The aim of present study was to develop different crystalline forms of metaxalone in order to overcome these drawbacks and consequently enhance its dissolution and bioavailability. For this purpose two different approaches were adopted namely cocrystallization and melt sonocrystallization. Cocrystals of metaxalonesaccharin and metaxalone-lactic acid were prepared, with saccharin and lactic acid as coformers, by using solvent evaporation method. Melt sonocrystallization technique was applied for preparation of melt sonocrystallized form of metaxalone. The percentage yield, melting point, particle size, flow property, crystallinity index, solubility and dissolution profiles of developed crystals were evaluated and compared with original metaxalone. Further the developed forms of metaxalone were characterized by differential scanning calorimetry, fourier transform infrared spectroscopy, X-ray diffraction and scanning electron microscopy. The sharp peak of differential scanning calorimetry thermograms and fourier transform infrared spectroscopy spectras indicate towards the formation of crystalline forms with retention of characterstic functionality of original metaxalone. The particle size and crystallinity index of the developed metaxalone crystals were significantly reduced in comparison to the original form. In addition, there was significant enhancement in the flow and compressibility of developed crystals, as indicated by the value of angle of repose, Carr's index and Hausner ratio. The solubility of prepared crystals was much higher as compared to metaxalone and progressively increased in the following order: metaxalonesaccharin<metaxalone-lactic acid<melt sonocrystallized form of metaxalone. The dissolution studies revealed that $87.08,92.79$ and $99.79 \%$ of the drug was released within $30 \mathrm{~min}$ from metaxalone-saccharin, metaxalone-lactic acid and melt sonocrystallized form of metaxalone crystals, respectively compared to $67.63 \%$ release from the original metaxalone drug. Further maximum solubility and faster dissolution was observed with melt sonocrystallized form of metaxalone as compared to metaxalone-lactic acid and metaxalone-saccharin. On the basis of this it can be concluded that solvent evaporation method and melt sonocrystallization techniques are promising techniques that may afford crystals with improved flow property, improved solubility and dissolution.

Key words: Metaxalone, melt sonocrystallization technique, solvent evaporation technique, cocrystals, solubility enhancement

Drug substances that are usually incorporated in dosage forms as a solid material are either in crystalline or amorphous form and selection of the appropriate polymorphic form of the drug is of utmost importance to ensure optimal solubility and absorption characteristics. Solubility is a key decisive factor in the formulation of dosage forms because drug molecules that are poorly soluble may lead to slow dissolution rates, insufficient and inconsistent systemic drug blood

*Address for correspondence E-mail: qaws200801@163.com levels and consequent sub-optimal efficacy ${ }^{[1]}$. About $40 \%$ of drugs available in the market and administered as oral tablets have poor aqueous solubility. In the This is an open access article distributed under the terms of the Creative
Commons Attribution-NonCommercial-ShareAlike 3.0 License, which
allows others to remix, tweak, and build upon the work non-commercially,
as long as the author is credited and the new creations are licensed under
the identical terms

Accepted 23 December 2020

Revised 22 September 2020

Received 04 August 2020

Indian J Pharm Sci 2020;82(6):974-983 
research and development pipeline, about 80-90\% of the new drug molecules fail to qualify for clinical trial stage due to poor aqueous solubility ${ }^{[2]}$. Thus, solubility enhancement of drugs is a major challenging aspect during the development of formulation strategies. Despite this distressing scenario, solubility problems can be overcome by either physicochemical modification of drug or by formulation of the Active Pharmaceutical Ingredient (API) into special dosage forms. Physicochemical modification can be achieved by application of crystal engineering to form either solvates, hydrates, polymorphs, cocrystals or cyclodextrin inclusion complexes and melt sonocrystallized (MSC) forms. Alternatively, special dosage forms such as solid dispersions, self-emulsifying formulations or liquid filled capsules may be developed to improve the dissolution profile of drug. The formation of cocrystals of drugs with coformers, with unique physicochemical properties, by using different techniques has been reported and confirmed by several scientists ${ }^{[3-9]}$. For the current work saccharine and lactic acid were selected to be used as coformers.

Saccharin (SACC) is an artificial sweetener which is commonly used as a coformer in cocrystallization. It has been reported in several studies to increase the solubility of drugs when used coformers ${ }^{[10-12]}$. Besides, lactic acid (LA) was used in the study because many carboxylic acids have been used historically as salt formers with drug substances, and are considered pharmaceutically acceptable. Several researchers have reported enhanced solubility of substances, cocrystallized with lactic acid $^{[13,14]}$. It was envisaged that the bioavailability issue of drugs due to poor aqueous solubility may be resolved by cocrystallization with SACC and LA. Besides both appear in the list of Generally recognized as safe (GRAS) and have been applied extensively as coformers in solvent evaporation technique.

Metaxalone (MTX) is an oxazolidin-2-one derived centrally acting muscle relaxant drug, available for relieving discomforts associated with acute, painful musculoskeletal conditions. MTX is commercially available in the form of conventional oral tablets but its utility is limited because of its low efficacy due to low aqueous solubility (category of Biopharmaceutics classification system (BCS) class-II). To achieve the desired drug blood levels high doses of drugs are required at frequent intervals of time, which leads to side effects and also patient non-compliance ${ }^{[15-17]}$. In order to enhance the therapeutic efficacy of the drug, MTX has been modified physicochemically to improve its solubility. This has been achieved by forming different crystalline forms of the drug by using various solvents/ excipients or using different techniques to yield crystals with unique physicochemical properties. The objective of the present study was to prepare cocrystal of MTX with coformer such as: SACC and LA using solvent evaporation technique and MSC form by using melt MSC technique in order to improve the physicochemical properties like flow properties, solubility, dissolution etc., and hence improved bioavailability.

\section{MATERIALS AND METHODS}

MTX was obtained as a gift sample from Sun Pharmaceutical (P) Ltd., Mumbai, India. SACC, LA, Methanol and Ethyl alcohol were procured from Central Drug Laboratories (P) Ltd., New Delhi. Liquid paraffin oil was obtained from S.D. Fine Chem. Ltd. Mumbai, India. Other chemicals and reagents used were of analytical grade.

\section{Preparation of Metaxalone-Saccharin (MTX- SACC) and Metaxalone-Lactic acid (MTX-LA) crystals by using solvent evaporation technique:}

The cocrystals of MTX with coformer such as: SACC and LA were prepared by using solvent evaporation technique ${ }^{[18,19]}$ with some modifications. MTX and coformer such as: SACC and LA were mixed in a ratio of 1:0.5 separately and completely dissolved in ethyl alcohol. The mixture was kept in water bath at a temperature at $80^{\circ}$ in order to obtain clear solution. The clear solution was then allowed to cool in an ice bath for around $5 \mathrm{~h}$ for complete crystallization. The crystals were collected by filtration through a Whatman filter paper, dried at room temperature for $24 \mathrm{~h}$ and finally stored in a desiccator for further use.

\section{Preparation of melt sonocrystallized (MTX-MSC) form of MTX crystals by MSC technique:}

The MSC form of drug was prepared using the MSC technology ${ }^{[3]}$. The pure form of MTX $(1 \mathrm{~g})$ was melted in a test tube on a paraffin oil bath maintained at $200^{\circ}$ temperature. Molten mass was poured into a beaker containing $50 \mathrm{ml}$ of double distilled water maintained at $25^{\circ}$ and sonicated for 4 min using probe ultrasonicator at a frequency of $33 \pm 3 \mathrm{kHz}$ at $80 \%$ amplitude with the power rating of 60 watts. The product obtained after solidification of dispersed droplets was separated by filtration using Whatman filter paper and dried at room temperature overnight and stored in a desiccator. 


\section{Characterization of MTX-SACC, MTX-LA cocrystal and MTX-MSC crystalline form of MTX:}

\section{Determination of percentage product yield}

The dried crystalline products of MTX were weighed individually (practical yield) and percentage yield of each product was calculated by the following equation ${ }^{[20]}$ :

Percentage yield $=($ Practical yield $/$ Theoretical yield )$\times 100$. The value of theoretical yield was taken as the total weight of the MTX and SACC, MTX and LA, and only MTX, in case of MTX-SACC, MTX-LA and MTX-MSC crystals, respectively.

\section{Determination of melting point}

The melting point of original form of MTX and developed crystalline forms of MTX was determined by capillary fusion method initially and subsequently the results were supplemented with Differential scanning calorimetry (DSC) studies carried out for all the three samples. Powder sample was filled in a capillary tube up to $3 \mathrm{~mm}$ height and placed in the melting point apparatus and a maximum temperature was set to make a quick measurement of melting point. The temperature at which sample got melted was observed with the help of magnifying lens and noted.

\section{UV-spectrophotometric analysis}

The UV-spectrophotometric analysis was performed according to method followed by Rao et al. 2011[21]. Weighed amounts $(25 \mathrm{mg}$ ) of MTX and each crystalline form of MTX were taken in $25 \mathrm{ml}$ of volumetric flask with minimum quantity of methanol, separately and volume was made up to $25 \mathrm{ml}$ with double distilled water. The sample was scanned using a UVspectrophotometer in a wavelength range of $200 \mathrm{~nm}$ to $400 \mathrm{~nm}$ and UV absorption maxima of each sample were observed.

\section{Differential scanning calorimetry (DSC)}

Thermal behavior of developed crystalline forms and original MTX was estimated using a differential scanning calorimeter (Labsys Evo Setaram Instruments, Model No.: D6492) instrument equipped with an intracooler. An indium standard was used to calibrate the temperature and enthalpy scale. The samples were hermetically sealed in aluminum pans and heated at a constant rate of $10 \% \mathrm{~min}$, over a temperature range of $0-300^{\circ}$. An inert atmosphere was maintained by purging with nitrogen at a flow rate of $40 \mathrm{ml} / \mathrm{min}$.

\section{Fourier Transform Infrared (FTIR) Spectroscopy}

FTIR spectra's were recorded using an FTIR Alpha with ECO ZnSe ATR (Bruker). The samples were placed with the help of capillary tube on the crystal surface ensuring good contact and scanned in the range of 4000 to $400 \mathrm{~cm}^{-1}$ at a resolution of $4 \mathrm{~cm}^{-1}$. The obtained frequency bands of developed crystal samples were compared with that of original form of MTX.

\section{Powder X-Ray diffraction (PXRD) spectroscopy}

The PXRD patterns of MTX and developed crystalline forms of MTX were obtained with an X-ray diffractometer (Mini-flex, Rigaku, Japan) under following conditions: $\mathrm{Cu} \mathrm{K} \alpha$ radiation (generated radiation at $1.54206 \AA$ Á wavelength); current $30 \mathrm{~mA}$; voltage $40 \mathrm{kV}$; scanning range $0-50^{\circ}$; scanning rate $2 \%$ min. The obtained data was used to calculate Crystallinity index, using equation ${ }^{[19,22]}$. Crystallinity index $(\%)=\left(\mathrm{I}_{020}-\mathrm{I}_{\text {am }} / \mathrm{I}_{020}\right) \times 100$, where, $\mathrm{I}_{020}$ is intensity at $22.5^{\circ}$ and $I_{a m}$ is lowest $2 \theta$ value near $18^{\circ}$.

\section{Surface morphology and particle size determination}

Surface and shape characteristics of original MTX and the developed MTX crystals were evaluated by using the Scanning electron microscope (SEM) technique ${ }^{[23]}$. Powder samples were coated uniformly with gold after fixing the samples in individual stubs and examined by SEM (model JSM-060, Jeol Ltd., Tokyo, Japan). Each photograph was saved as a jpeg file. From the photographs, particle axes were measured using Motic Images Advanced 3.2 software selecting images with no overlapping and no border out of the picture frame were chosen for axes measurements. The major axes length (L) and width (W) of each particle were measured and entered into a Microsoft excel spreadsheet. The mean values of three lines drawn on the particle for each axis were taken as the real $\mathrm{L}$ and $\mathrm{W}$ values of that particle in micrometers considering the scale of the image. The area (A) of the particle was calculated on the basis of the measured $\mathrm{L}$ and $\mathrm{W}$, as shown in given equation ${ }^{[24]}$ : $\mathrm{A}=(\pi \mathrm{LW}) / 4$

\section{Rheological characterization}

Flow properties were evaluated by determining the various rheological indicators namely: poured density, tapped density, Carr's compressibility index (\%), Hausner ratio (HR) and angle of repose ${ }^{[25]}$. The angle of repose was measured by using fixed funnel method and tapped density was measured by the tapping method ${ }^{[26]}$. For the determination of tapped density, $1 \mathrm{~g}$ of powder 
samples was poured into $10 \mathrm{ml}$ graduated measuring cylinder and the poured volume was noted down. The cylinder was then subjected to 100 tapping until the change in volume became constant, using tapped density apparatus $\left(\mathrm{HICON}^{\circledR}\right.$, New Delhi) and tapped volume was noted down. The tapped density was then obtained by dividing the weight of sample by the final volume (in cubic centimetre) of the sample contained in the cylinder. The Carr's compressibility index was computed according to the following equation ${ }^{[27]}$ :

Compressibility Index $=$ (Tapped density-poured density)/Tapped density $\times 100$

HR was determined by comparing the tapped density to the fluff density using the following equation ${ }^{[28]}$ : Hauser ratio=tapped density/poured density

\section{Equilibrium solubility study}

To determine the solubility of MTX, MTX-SACC, MTX-LA and MTX-MSC, saturation solubility measurements was carried out by using shake flask method $^{[22]}$. An excess amount of MTX, MTX-SACC, MTX-LA and MTX-MSC were separately added to $10 \mathrm{ml}$ of double distilled water $(\mathrm{pH} \mathrm{6.8)}$ in conical flasks and maintained on water bath shaker $37^{\circ} \pm 5^{\circ}$ for a period of $72 \mathrm{~h}$ until equilibrium was attained. The solutions were then centrifuged at $7000 \mathrm{rpm}$ for $10 \mathrm{~min}$ and supernatants were filtered using Whatman filter paper \#1, suitably diluted and amount of drug analysed spectrophotometrically using UV Spectrophotometer (Shimadzu UV 1800, Kyoto, Japan).

\section{Dissolution study}

Dissolution studies were performed using the United States Pharmacopeia (USP) 24 type-II dissolution test apparatus. MTX and the developed crystals
(20 $\mathrm{mg}$ ) were separately placed in the dissolution vessels containing $900 \mathrm{ml}$ of double distilled water $\mathrm{pH}$ 6.8 , previously maintained at $37 \pm 0.5^{\circ}$ temperature and stirred at $100 \mathrm{rpm}$. Periodically, samples were collected and replaced with fresh dissolution medium at specific time intervals $(0,5,10,15,20,25$ and $30 \mathrm{~min}$ ). After filtration through $0.45 \mu \mathrm{m}$ Whatman filter paper, samples were analyzed by UV-Visible spectrophotometer (Shimadzu UV 1800, Kyoto, Japan) [29].

\section{RESULTS AND DISCUSSION}

Different cocrystals of MTX that is MTX-SACC and MTX-LA were formulated by using solvent evaporation technique and MSC form was developed by MSC technique. The prepared crystals were characterized for their properties as listed in Table 1. The process yield of MTX crystal products was in the range of 73.03-82.52\% with highest yield being for MTXLA as compared to MTX-SACC and MTX-MSC. The products were screened for crystals formation by melting point, UV Spectrophotometry, DSC and FTIR studies. The different crystalline forms MTX, MTXSACC, MTX-LA and MTX-MSC exhibited sharp melting points at a temperature of $124,121.75,120.3$ and $122.83^{\circ}$ respectively. UV absorption maximum for different forms was found to be $278.9 \mathrm{~nm}$ for MTX, $280.18 \mathrm{~nm}$ for MTX-SACC, $275.6 \mathrm{~nm}$ for MTX-LA and 279.4 for MTX-MSC. The changes in melting point and UV Absorption maxima are indicative of possible transformation in the crystal structure due to cocrystallization and melt-sonocrystallization.

Further DSC and FTIR studies supported the above observations and DSC thermogram of different crystalline forms is shown in fig. 1. Original MTX exhibits a single endothermic peak at $126.08^{\circ}$

TABLE 1: COMPARATIVE DATA OF VARIOUS EVALUATION PARAMETERS OF DEVELOPED CRYSTAL FORMS AND ORIGINAL MTX

\begin{tabular}{lcccc}
\hline Parameters & MTX (Pure) & MTX-SACC & MTX-LA & MTX-MSC \\
\hline Yield $^{\mathrm{a}}(\%)$ & - & $73.03 \pm 0.646$ & $82.52 \pm 1.300$ & $80.46 \pm 0.982$ \\
Melting Point $^{\mathrm{a}}\left({ }^{\circ}\right)$ & $124 \pm 0.50$ & $121.75 \pm 0.410$ & $120.3 \pm 0.620$ & $122.83 \pm 0.743$ \\
UV Absorption Maxima $(\mathrm{nm})^{\text {Particle Size }}(\mu \mathrm{m})$ & 278.9 & 280.1 & 275.6 & 279.4 \\
Poured Density $^{\mathrm{a}}(\mathrm{g} / \mathrm{cc})$ & $92.12 \pm 1.183$ & $79.61 \pm 1.18$ & $39.56 \pm 1.24$ & $35.78 \pm 0.82$ \\
Tapped Density $^{\mathrm{a}}(\mathrm{g} / \mathrm{cc})$ & $0.322 \pm 0.002$ & $0.552 \pm 0.0027$ & $0.666 \pm 0.003$ & $0.762 \pm 0.0039$ \\
Compressibility Index $^{\mathrm{a}}(\%)$ & $0.454 \pm 0.012$ & $0.661 \pm 0.016$ & $0.769 \pm 0.018$ & $0.847 \pm 0.018$ \\
Hausner Ratio $^{\mathrm{a}}$ & $29.00 \pm 3.000$ & $16.49 \pm 1.167$ & $13.39 \pm 1.039$ & $10.03 \pm 1.05$ \\
Angle of Repose $^{\mathrm{a}}($ Degree $)$ & $1.40 \pm 0.045$ & $1.19 \pm 0.015$ & $1.15 \pm 0.014$ & $1.11 \pm 0.013$ \\
Crystallinity Index $(\%)^{\text {Solubility }}{ }^{\mathrm{a}}\left(\mathrm{mg} \mathrm{ml}^{-1}\right)$ & $47.67 \pm 1.263$ & $34 \pm 0.734$ & $32.00 \pm 0.678$ & $26.00 \pm 0.525$ \\
\hline
\end{tabular}

aExperiments were performed in triplicate, Mean \pm standard deviation of three determination. MTX is Metaxalone, SACC is Saccharin, LA is the lactic acid, MSC is melt sonocrystallized 

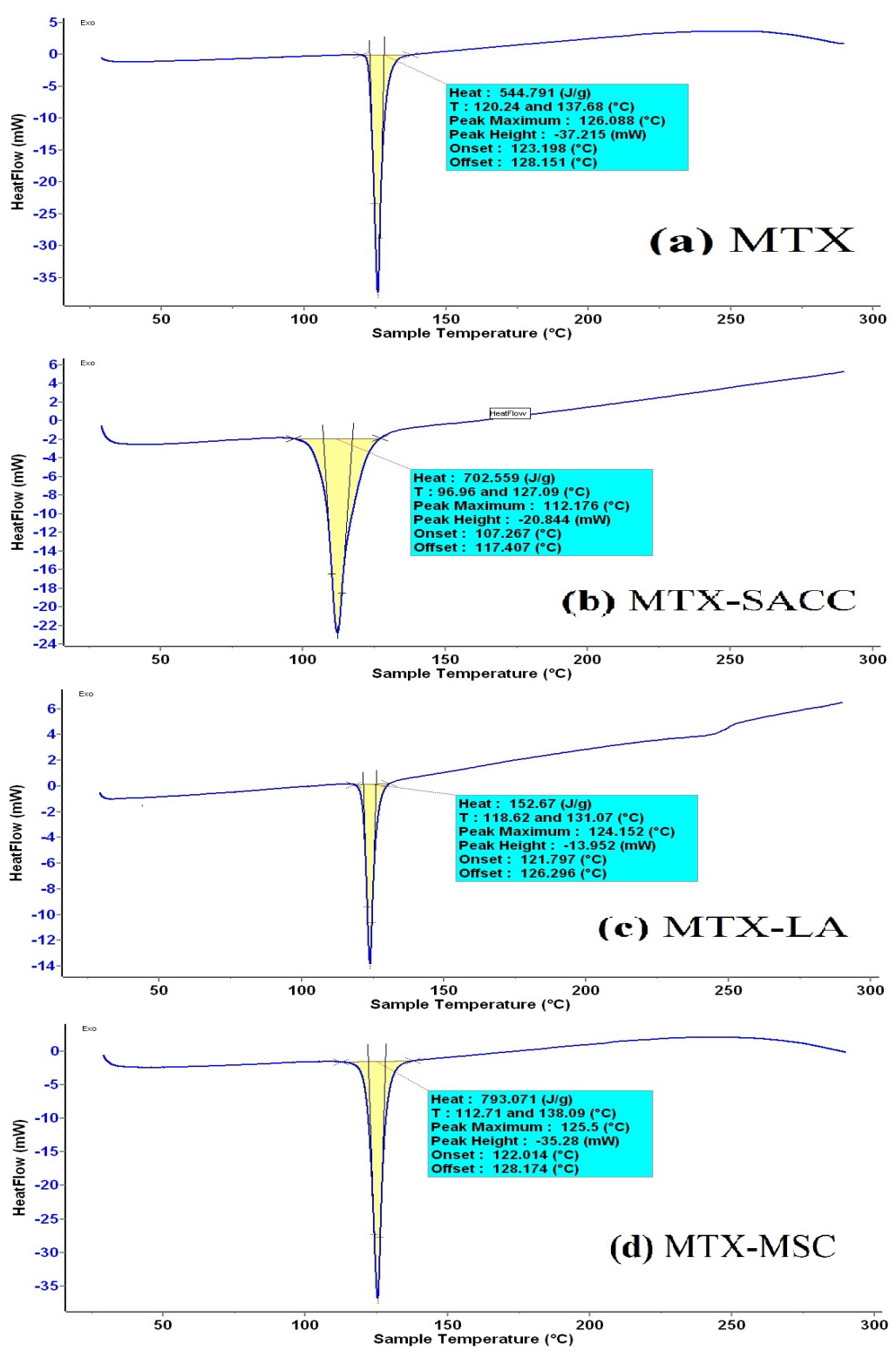

Fig. 1: DSC thermograms of different crystal forms of MTX: MTX (a), MTX-SACC (b), MTX-LA (c) and MTX-MSC (d)

and cocrystals of MTX-SACC exhibited a single endothermic peak at $112.17^{\circ}$ which could be attributed to cocrystals formation. Similarly, MTX-LA cocrystals exhibited a single sharp endothermic peak at $124.15^{\circ}$ which can be attributed to cocrystallization of MTX with lactic acid. The MSC forms exhibited a sharp single endothermic peak at $125.5^{\circ}$ which is comparable to the endothermic peak obtained with original MTX at $126.08^{\circ}$. The minor difference in the two is attributed to change in crystal habit of MTX.

Vibrational spectroscopy is a preliminary tool to differentiate between the starting materials and the final products. In this case the new crystals formation is accompanied by significant changes in the vibrational frequencies between the prepared crystals and the active drug moiety. MTX exhibits two sharp characteristic
Infrared (IR) bands at $3265.53 \mathrm{~cm}^{-1}$ (Strong amide $\mathrm{N}-\mathrm{H}$ vibration) and at $1724.93 \mathrm{~cm}^{-1}(\mathrm{C}=\mathrm{O}$ stretching $)$ as seen in fig. 2a. The FTIR spectra of MTX-SACC form (fig. 2b) exhibited sharp absorption band at $1640.45 \mathrm{~cm}^{-1}$ and $1248.97 \mathrm{~cm}^{-1}$ attributed to N-H bending and C-N stretching of SACC molecule. Additionally a sharp stretching band at $1143.60 \mathrm{~cm}^{-1}$ indicates the presence of S-O group of SACC, whereas the typical IR band for MTX were observed at $3276.85 \mathrm{~cm}^{-1}$ ( $\mathrm{N}-\mathrm{H}$ stretching) and $1729.68 \mathrm{~cm}^{-1}(\mathrm{C}=\mathrm{O}$ stretching). In case of MTX-LA cocrystal form, shoulder peak of $\mathrm{N}-\mathrm{H}$ (Metaxalone $\mathrm{N}-\mathrm{H}$ stretching) with a broad peak of $\mathrm{OH}$ group shown in fig. 2c indicate involvement of hydrogen bonding between MTX and lactic acid during cocrystallization. The spectra of MTX-MSC exhibited amplification of $\mathrm{C}=\mathrm{O}$ band at $1727.60 \mathrm{~cm}^{-1}$ (fig. 2d) which could be indicative of breakage of $\mathrm{C}=\mathrm{O}$ bond of 

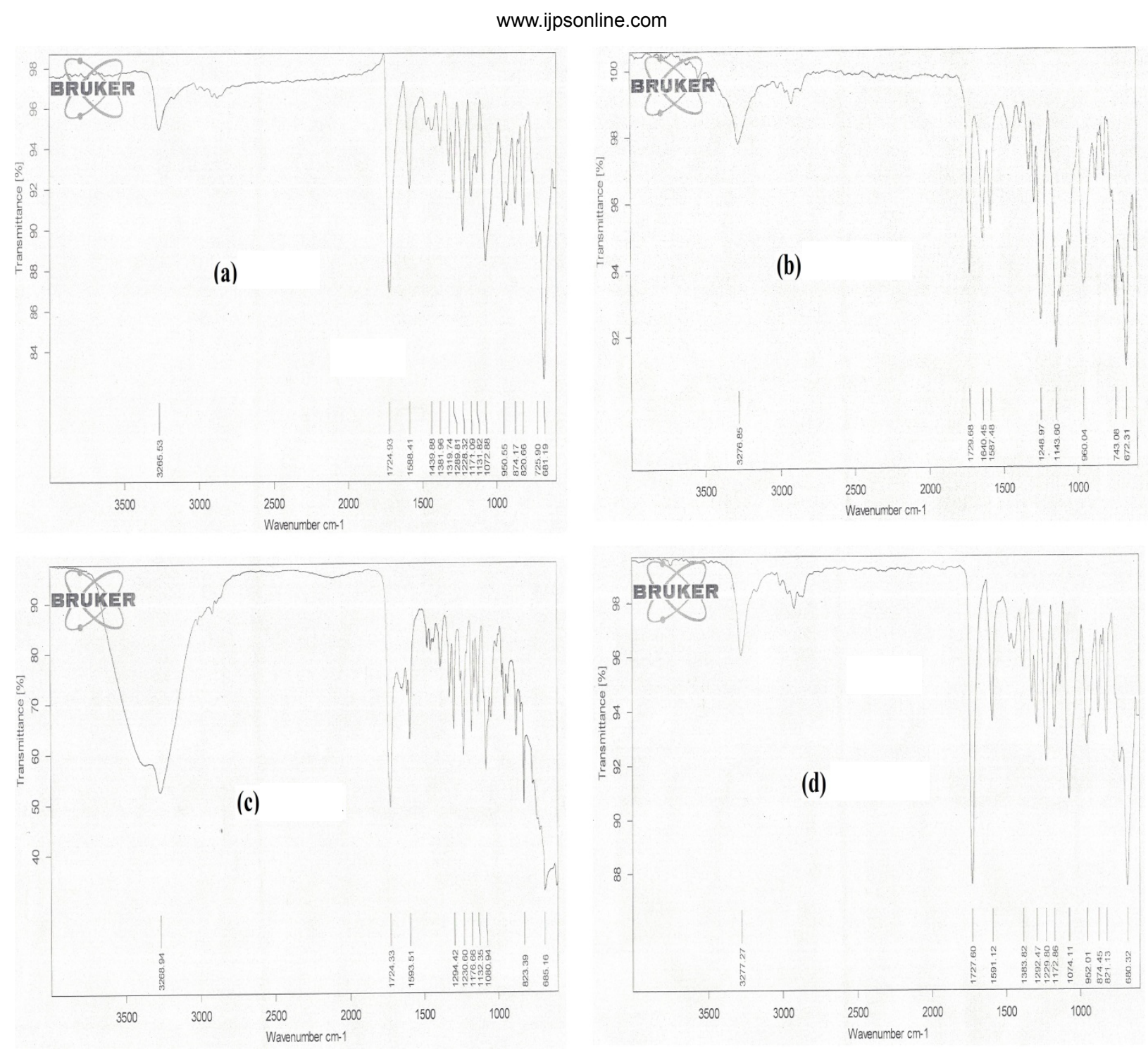

Fig. 2: FTIR spectra of original MTX and developed crystal forms: MTX (a), MTX-SACC (b), MTX-LA (c) and MTX-MSC (d)

metaxalone during melting. The spectras of developed crystalline forms when compared to that of original MTX clearly imply the formation of novel crystals.

The crystallinity index and the structural features of developed crystalline forms were revealed by PXRD studies. Crystallinity index (Table 1) was calculated by the formula as shown by Lin et al. 2014 ${ }^{[19]}$ and Kumar et al. 2013 ${ }^{[22]}$. Fig. 3 shows the PXRD patterns of MTX (a), MTX-SACC (b), MTX-LA (c) and MTXMSC (d). The X-ray diffractograms of MTX drug showed sharp and well-defined diffraction peaks in the $2 \theta$ range of $0-50^{\circ}$ indicating that the unprocessed drug was crystalline in nature as shown in fig. 3a. Apparently, the PXRD patterns for the developed crystals of MTX were significantly different from the original MTX. However, the relative intensities of the diffraction peaks were also modified. This could be attributed to the markedly different crystal habits of the MTX-SACC, MTX-LA and MTX-MSC. The underlying cause could be alterations in the relative abundance of the planes exposed to the X-ray source producing variations in the intensities of peak or may be due to differences in crystal sizes ${ }^{[18,30]}$. In order to quantitate the reduction in crystallinity, the diffractograms were used to calculate the crystallinity index. The crystallinity index of original MTX was found to be $93.44 \%$, while MTX-SACC, MTX-LA and MTX-MSC showed a crystallinity index of $82.26 \%, 76.52 \%$ and $74.35 \%$, respectively. The lowest crystallinity index was observed in MTX-MSC followed by MTX-LA and MTX-SACC. The change in crystallinity was also visualized by scanning electron microscopy (SEM) photographs. The results of PXRD data strongly confirm the cocrystals formation between MTX and SACC, LA and also confirm sonocrystallized form of MTX.

Crystal morphology and particle size influence various pharmaceutical parameters such as flow ability, packing, compaction, compressibility, solubility and 

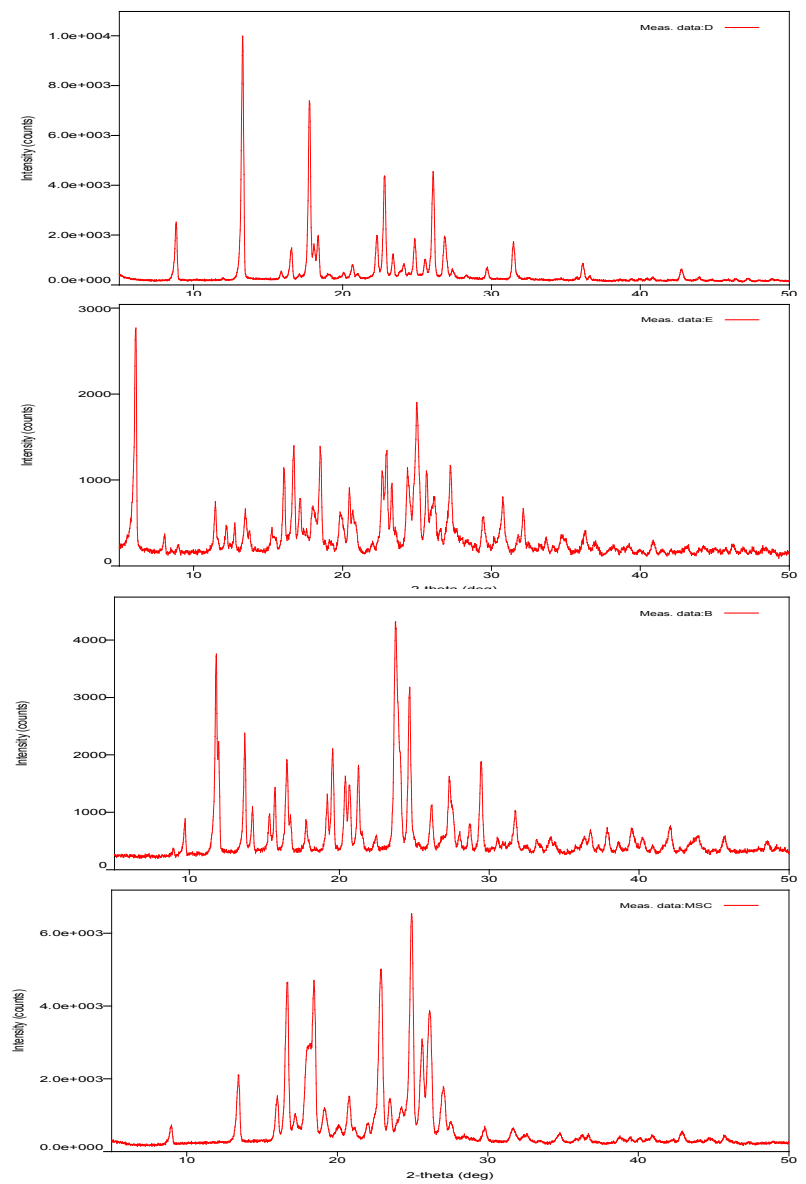

Fig. 3: PXRD pattern of different crystalline forms of MTX: MTX (a), MTX-SACC (b), MTX-LA (c) and MTX-MSC (d) dissolution characteristic of drug powder ${ }^{[3]}$. SEM images as shown in fig. 4 were used to visualize the morphology of original and developed crystal forms of the drug (MTX). The original form of the drug (MTX) was large in size with uneven surface and needle/ rod shape structure as shown in fig. 4a whereas the developed crystal forms were clusters/aggregates of small crystals with irregular pores of various shapes and sizes when observed at lower magnifications. The MTX-SACC cocrystal were observed to have lopsided, uniform, plane surface and straight boundaries in their crystalline structure and also had pitted and shrunken areas on their surface as represented in fig. $4 \mathrm{~b}$. Fig. 4c shows the MTX-LA cocrystal particles which appeared to be smaller, uniform and much regular in shape and surface. The MSC form of MTX was fine, sharp edged, smooth surfaced with abundant planes and a porous crystalline structure (fig. 4c). This could be due to sonication of the molten mass, which might have created cracks and led to an increase in the intra particulate porosity of MTX-MSC. The decrease in surface roughness on sonocrystallization has also been reported by El-Kamel, 2008 and by Manish et al., $2005^{[3]}$. High magnification images also confirmed that smaller particles with smooth surfaces and variable shapes were observed in developed crystals form of MTX. The smoothness of the particle surface can be (a)
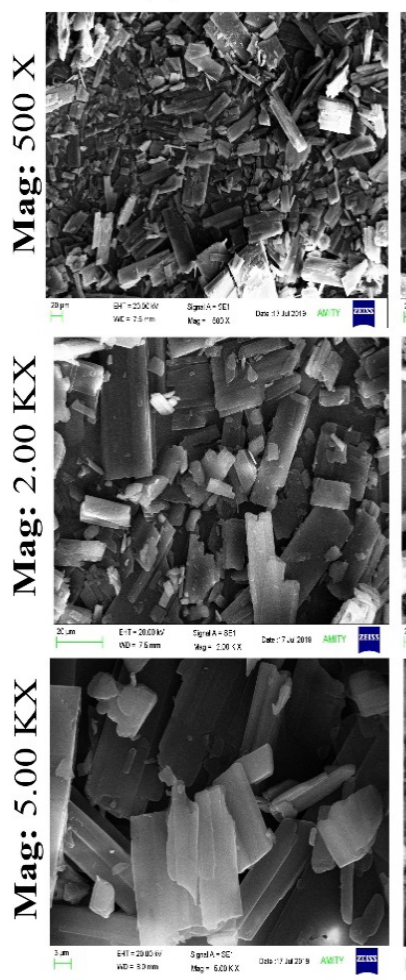

(b)
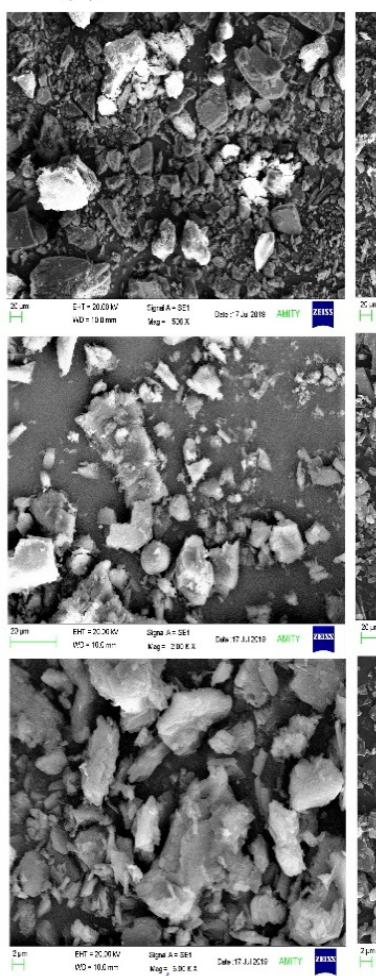

(c)
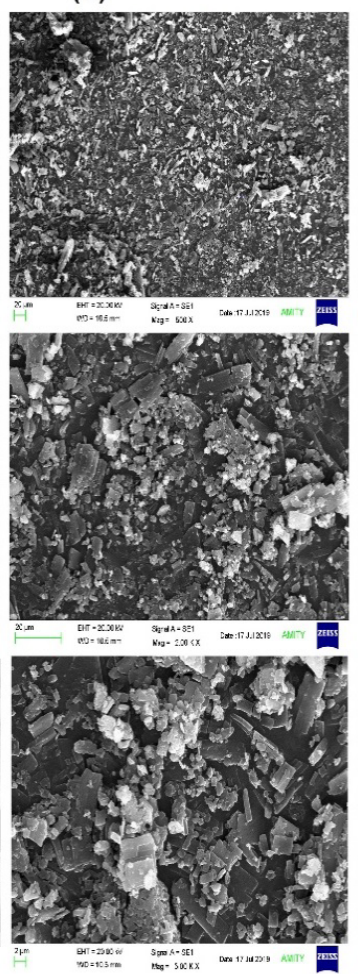

(d)
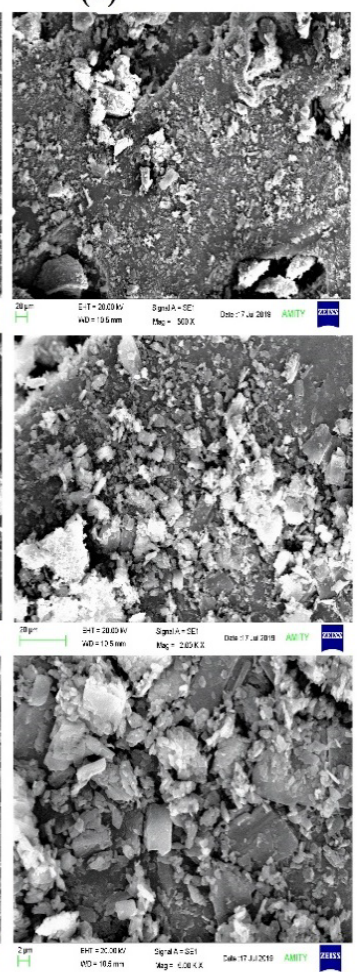

Fig. 4: SEM images of original form and prepared crystal forms of MTX: MTX (a), MTX-SACC (b), MTX-LA (c) and MTX-MSC (d) at different magnifications 
an added advantage to facilitate the flow of powder and this is well evident from the obtained values of angle of repose. A magnified view, fig. 2 clearly depicts the changes in crystal structure including development of cracks in the crystals, decrease in particle size and a reduction in surface roughness in developed crystals of MTX. Powder surface roughness makes the particles prone to sticking that was also claimed by Rasenack and Muller, 2002 $2^{[31]}$ and consequently, prepared cocrystals and MSC forms of MTX would be expected to have less sticking properties than that of the original form of MTX. Thus, solvent evaporation method and sonocrystallization can be considered as an effective tool to affect surface morphology and structure of crystalline forms of drug.

The particle size analysis revealed reduction in particle size from $92.12 \pm 1.18 \mu \mathrm{m}$ (MTX) to $79.61 \pm 1.18$ (MTX-SACC) followed by 39.56 \pm 1.24 (MTX-LA) and $35.78 \pm 0.82 \mu \mathrm{m}$ (MTX-MSC), sub sequentially may have led to an increase in surface area that could have resulted in enhanced solubility. The reduction in particle size can be attributed to the intensified micro mixing that led to enhanced mass transfer and diffusion between solvent and crystallization magma, which induces prompt high levels of supersaturation that result in rapid nucleation and increase in the number of primary nuclei, so the amount of solute depositing on each primary nucleus decreases and leads to decreased final size of crystals.

The surface morphology and particle size in turn influences the flow characteristics of powder crystals that can be assessed by the angle of repose determination. Flow property is important in order to achieve weight uniformity in tablet production. Rheological data for MTX and prepared crystal forms of MTX are shown in Table 1. The calculated values of angle of repose for MTX, MTX-SACC, MTX-LA and MTX-MSC were $47.67 \pm 1.263,34 \pm 0.734,32.00 \pm 0.678$

TABLE 2: PERCENTAGE DRUG RELEASE PROFILE OF ORIGINAL AND DEVELOPED CRYSTALLINE FORMS OF MTX

\begin{tabular}{lcccc}
\hline Time & \multicolumn{4}{c}{ Percentage Drug Release $^{\mathrm{b}}$} \\
\cline { 2 - 5 } (Min) & MTX (Pure) & MTX-SACC & MTX-LA & MTX-MSC \\
0 & 0.00 & 0.00 & 0.00 & 0.00 \\
5 & $22.56 \pm 0.91$ & $45.28 \pm 0.61$ & $51.93 \pm 0.89$ & $58.05 \pm 0.61$ \\
10 & $36.74 \pm 0.90$ & $55.92 \pm 1.15$ & $65.32 \pm 1.56$ & $68.81 \pm 1.42$ \\
15 & $42.85 \pm 1.24$ & $63.87 \pm 1.53$ & $72.81 \pm 1.49$ & $79.04 \pm 1.12$ \\
20 & $50.03 \pm 1.52$ & $70.82 \pm 1.93$ & $80.46 \pm 1.81$ & $85.93 \pm 1.76$ \\
25 & $61.87 \pm 1.36$ & $78.94 \pm 1.45$ & $87.53 \pm 1.36$ & $94.71 \pm 1.28$ \\
30 & $67.63 \pm 1.45$ & $87.08 \pm 0.74$ & $92.79 \pm 1.45$ & $99.79 \pm 1.54$ \\
\hline
\end{tabular}

${ }^{\text {bAll data were expressed as mean } \pm \text { S.D, S.D is standard deviation for }}$ observations $\mathrm{n}=3$ and $26.00 \pm 0.525$ degree, respectively. It was clearly noticeable that the prepared MTX crystals had better flow properties than the original MTX, with an angle of repose $<40^{\circ}$. The poured density and tapped density of MTX-SACC, MTX-LA and MTX-MSC were higher than the original form of MTX. The most important factor affecting the poured and tapped density of a powder and its flow properties are void spaces and inter particulate interaction including friction and adhesion. The calculated values of compressibility index of newly developed crystal forms of MTX was found to be significantly lower than that calculated for the MTXdrug, indicating better compressibility features of developed crystals as compared to original form of MTX. Thus, crystals with lower values of Carr's compressibility index can be considered as more acquiescent to tablet production steps. HR is another parameter used to evaluate the powder flow ability, prepared crystals of MTX exhibited HR value lying between 1.11 to 1.19 which were lower than 1.40 of original MTX this again proves superior flow properties of the developed crystals of MTX as compared to original form of the drug. It can hence be summarized that the smaller values of angle of repose, Carr's compressibility index and HR are consistent with developed MTX crystals having relatively improved flow properties. The same is expected, due to a decreased inter-particulate interaction owing to decrease in surface roughness that was visualized and detected by SEM examination (fig. 4). In addition, it has been reported that the smooth surface of the particles results in smaller friction ${ }^{[32]}$ due to decreased contact points between particles and hence enhanced flow. This was again in agreement with the current results, since the treated MTX that is the newly formed crystals of MTX which had smoother surface showed better flow properties.

From the result of equilibrium solubility studies as shown in Table 1, it was observed that, the new crystal forms of MTX drug exhibited enhanced solubility in double distilled water as compared to the original form of drug. Solubility of pure MTX was found to be $0.1589 \pm 0.0056 \mathrm{mg} / \mathrm{ml}$, whereas for the developed MTX crystals, it was found that solubility increased to $0.1891 \pm 0.0042, \quad 0.3406 \pm 0.0087$ and 0.3824 $\pm 0.0034 \mathrm{mg} / \mathrm{ml}$ for MTX-SACC, MTX-LA and MTX-MSC, respectively (fig. 5). The increase in solubility may also be due to a decrease in particle size, consequently increase in surface area as observed in particle size analysis results were also reported by Manish et al., 2005 ${ }^{[3]}$ and Tripathi et al., 2010 ${ }^{[33]}$ and the fine cracks/pores were also observed in SEM analysis 


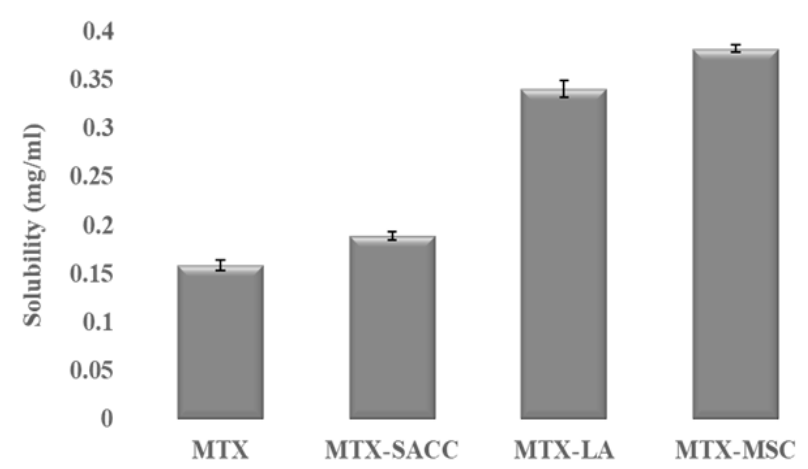

Fig. 5: Bar diagram depicting solubility data of original and developed crystal forms of MTX

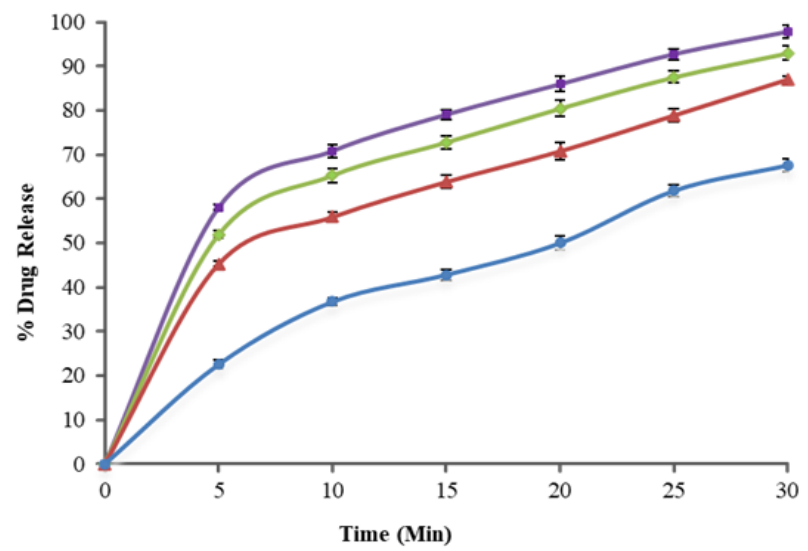

Fig. 6: Percentage drug release pattern of MTX from original and developed crystals

MTX- MSC (--), MTX-LA $(-\downarrow)$, MTX-SAC (- - -), $\operatorname{MTX}(-\bullet-)$

on the crystals particle surfaces that facilitates solvent ingress and consequently solubility enhancement ${ }^{[3]}$.

Dissolution profile of MTX drug and different crystals of MTX has been shown in in Table 2 and fig. 6, where it is understandable that crystals prepared with different coformers using different techniques resulted in changes in the drug release profile of MTX. Unprocessed MTX shows a release of $67.63 \pm 1.45 \%$ drug in $30 \mathrm{~min}$. Compared to pure MTX drug, the dissolution rate of developed crystals has been significantly increased to $87.08 \pm 0.74,92.79 \pm 1.45$ and $99.79 \pm 1.54 \%$ in $30 \mathrm{~min}$ for MTX-SACC, MTX-LA and MTX-MSC, respectively. The dissolution studies showed that the release rates of MTX-SACC and MTX-LA cocrystals prepared by using solvent evaporation were higher as compared to the pure drug. This may be attributed to the decrease in the drug crystallinity (shown in Table 1) and also, the significant drug particle size reduction achieved, which have direct bearing on dissolution rates. Furthermore, the MTX-MSC form showed highest, rather almost complete release $(99.79 \%)$ in $30 \mathrm{~min}$ because it is prepared by MSC technique and Ultrasonic treatment has caused significant changes in the dissolution rate. This may be attributed to increase in surface area due to porous nature of the crystals obtained after ultrasonic treatment.

The study utilizes crystal engineering techniques such as solvent evaporation and MSC for processing the poorly water-soluble (BCS class-II) MTX drug to improve its primary and secondary pharmaceutical characteristics. The results indicate SACC and LA can form cocrystals with MTX via solvent-evaporation technique and also form MSC form of MTX by MSC technique. The poor solubility and dissolution profile of MTX was seen to have improved by forming cocrystals and MSC forms. Flow properties were found to improve due to decrease in the surface roughness leading to reduced cohesiveness and in turn improvement in compression characteristics due to changes in crystal habit. The present study revealed that as compared to other techniques, the MSC technique demonstrated remarkable improvement in flow properties, solubility and dissolution of MTX through the modification in particle size, degree crystallinity and surface topography. The processed MTX crystals are suitable and can be formulated into oral dosage forms.

\section{Acknowledgements:}

The authors are grateful to the Sun Pharmaceutical (P) Ltd., Mumbai, India for providing gift sample of MTX for this research work.

\section{Conflict of Interests:}

The authors declared no conflict of interest.

\section{REFERENCES}

1. Blagden N, de Matas M, Gavan PT, York P. Crystal engineering of active pharmaceutical ingredients to improve solubility and dissolution rates. Adv Drug Deliv Rev 2007;59(7):617-30.

2. Najar AA, Azim Y. Pharmaceutical co-crystals: Anew paradigm of crystal engineering. J Indian Inst Sci 2014;94(1):45-68.

3. Manish M, Harshal J, Anant P. Melt sonocrystallization of ibuprofen: Effect on crystal properties. Eur J Pharm Sci 2005;25(1):41-8.

4. Caira MR, Stieger N, Liebenberg W, Villiers MM, Samsodien $\mathrm{H}$. Solvent inclusion by the anti-HIV drug nevirapine: X-ray structures and thermal decomposition of representative solvates. Cryst Growth Des 2008;8(1):17-23.

5. Chadha R, Arora P, Saini A, Jain DS. Solvated crystalline forms of nevirapine: thermoanalytical and spectroscopic studies. AAPS PharmSciTech 2010;11(3):1328-39.

6. Lyn L, Sze H, Rajendran A, Adinarayana G, Dua K, Garg S. Crystal modifications and dissolution rate of piroxicam. Acta Pharm 2011;61(4):391-402.

7. Caira MR, Bourne SA, Samsodien H, Engel E, Liebenberg W, Stieger $\mathrm{N}$, et al. Co-crystals of the antiretroviral nevirapine: 
crystal structures, thermal analysis and dissolution behaviour. Cryst Eng Comm 2012;14(7):2541-51.

8. Goke K, Lorenz T, Repanas A, Schneider F, Steiner D, Baumann $\mathrm{K}$, et al. Novel strategies for the formulation and processing of poorly water-soluble drugs. Eur J Pharm Biopharm 2018;126:40-56.

9. Zhang X, Xing H, Zhao Y, Ma Z. Pharmaceutical dispersion techniques for dissolution and bioavailability enhancement of poorly water-soluble drugs. Pharmaceutics 2018;10(3):74.

10. Hickey MB, Peterson ML, Scoppettuolo LA, Morrisette SL, Vetter A, Guzman H, et al. Performance comparison of a cocrystal of carbamazepine with marketed product. Eur J Pharm Biopharm 2007;67(1):112-9.

11. Siswandi S, Rusdiana T, Levita J. Virtual screening of coformers for ketoprofen cocrystallization and the molecular properties of the co-crystal. J App Pharm Sci 2015;5(6):78-82.

12. Payghan SA. Preparation and Characterization of Molecular Complexes of Fenofibrate Cocrystal. Asian J Pharm 2018;11(4).

13. Stanton MK, Bak A. Physicochemical properties of pharmaceutical co-crystals: a case study of ten AMG 517 cocrystals. Cryst Growth Des 2008;8(10):3856-62.

14. Rajurkar VG, Sunil NA, Ghawate V. Tablet formulation and enhancement of aqueous solubility of efavirenz by solvent evaporation Co-Crystal technique. Med chem 2015;S2:21610444.

15. Witenko C, Moorman-Li R, Motycka C, Duane K, HincapieCastillo J, Leonard $\mathrm{P}$, et al. Considerations for the appropriate use of skeletal muscle relaxants for the management of acute low back pain. Pharm Ther 2014;39(6):427-35.

16. Vasanani PR, Patel L, Detroja C. Formulation and Development of Stable Metaxalone Nanosuspension Using 32 Factorial Design. Int J Pharm Sci Drug Res 2016;8(4):223-8.

17. Vuletic L, Khan MZ, Spoljaric D, Radic M, CetinaCizmek B, Filipovic-Grcic J. Development of a clinically relevant dissolution method for metaxalone immediate release formulations based on an IVIVC model. Pharm Res 2018;35(8): 163 .

18. Papageorgiou GZ, Bikiaris D, Karavas E, Politis S, Docoslis A, Park Y, et al. Effect of physical state and particle size distribution on dissolution enhancement of nimodipine/ PEG solid dispersions prepared by melt mixing and solvent evaporation. AAPS J 2006;8(4):E623-31.

19. Lin HL, $\mathrm{Wu} \mathrm{TK}$, Lin SY. Screening and characterization of cocrystal formation of metaxalone with short-chain dicarboxylic acids induced by solvent-assisted grinding approach. Thermochim Acta 2014;575:313-21.
20. Gupta PS, Sharma V, Pathak K. Melt sonocrystallized piroxicam for oral delivery: Particle characterization, solid state analysis, and pharmacokinetics. Expert Opin Drug Deliv 2013;10(1):17-32.

21. Rao NR, Bn AK, Laxmi A. Method validation of metaxalone drug by using UV spectroscopy. Int $\mathrm{J}$ Pharm Bio Sci 2011;1(3):284-302.

22. Kumar B, Sharma V, Pathak K. Effect of melt sonocrystallization on pharmacotechnical properties of paracetamol, indomethacin and mefenamic acid characterized by dynamic laser scattering and its impact on solubility. Drug Dev Ind Pharm 2013;39(5):687-95.

23. El-Kamel AH. Improvement of physicochemical and biopharmaceutical properties of flurbiprofen using melt sonocrystallization technique. Drug Dev Res 2008;69(1):3441.

24. Beyer WH. Handbook of mathematical sciences. 5th ed. West Palm Beach, Florida: CRC Press; 1978.

25. Sinko PJ. Martin's physical pharmacy and pharmaceutical sciences. 5th ed. Wolters Kluwer (India) Pvt Ltd., New Delhi, India; 2008. p. 533-59.

26. Banker G, Anderson R. Tablets. In: Lachman L, Lieberman HA, Kanig JL, editors. The theory and practice of industrial pharmacy. Philadelphia: Lea \& Febiger; 1987. p. 316-7.

27. Carr RL. Evaluating flow properties of solids. Chem Eng 1965;18:163-8.

28. Hausner HH. 1967. Friction conditions in a mass of metal powders. Int J Powder Met 1967; 3(4):7-13.

29. Rajalakshmi S, Pawar AP, Mali AJ, Bothiraja C. Crystal engineering of bioactive plumbagin using anti-solvent precipitation, melt solidification and sonocrystallization techniques. Mater Res Express 2014;1(2):025405.

30. Sridevi S, Diwan PV. Optimized transdermal delivery of ketoprofen using $\mathrm{pH}$ and hydroxypropyl- $\beta$-cyclodextrin as coenhancers. Eur J Pharm Biopharm 2002;54(2):151-4.

31. Rasenack N, Muller BW. Ibuprofen crystals with optimized properties. Int J Pharm 2002;245(1-2):9-24.

32. Xinde XU, Shanjing YA, Ning HA, Bin SH. Measurement and influence factors of the flowability of microcapsules with highcontent $\beta$-carotene. Chin J Chem Eng 2007;15(4):579-85.

33. Tripathi R, Biradar SV, Mishra B, Paradkar AR. Study of polymorphs of progesterone by novel melt sonocrystallization technique: A technical note. AAPS Pharm Sci Tech 2010;11(3):1493-8. 